\title{
Cognition and discourse production in Alzheimer's disease: using informative prompts
}

\author{
Lenisa Brandão ${ }^{1}$, Fátima Galiana Castelló ${ }^{2}$ Teun A. van Dijk², Maria Alice de Mattos Pimenta \\ Parente $^{1}$ and Jordi Peña-Casanova ${ }^{3}$
}

1 Universidade Federal do Rio Grande do Sul, Brazil.

2 Pompeu Fabra University, Spain.

3 Institut Municipal d’Investigació Mèdica, Spain.

\begin{abstract}
This article reports an investigation on the cognitive and linguistic mechanisms involved in knowledge management during discourse production of individuals with Alzheimer disease (AD). Two discourse variables were examined, incomplete propositions and repeated propositions. Differences between controls and participants with AD were found concerning the presence of incomplete propositions in a non-informative prompted task. Findings obtained with the informative prompted task suggested that knowledge management was more preserved in the early stage than in the later stage of AD. The majority of the neuropsychological data obtained in this study correlated with the presence of incomplete propositions. The discussion debated on whether this discourse deficit can be attributed to an impaired ability to manage knowledge provided in the context of communication. Keywords: discourse, prompts, cognition, knowledge, Alzheimer's disease.
\end{abstract}

Received 3 November 2009; received in revised form 11 December 2009; accepted 12 December 2009. Available on line 29 December 2009.

\section{Introduction}

Alzheimer's disease (AD) is clinically defined as a degenerative cerebral pathology which affects cognitive abilities. It is characterized by multiple deficits that compromise the individuals' mental and social functioning. Some of the most salient impairments that arise with the disease relate to discourse disorders. Discourse declines significantly in the course of $\mathrm{AD}$, becoming increasingly

Lenisa Brandão, Department of Developmental Psychology, Universidade Federal do Rio Grande do Sul (UFRGS), Porto Alegre, RS, Brazil. Fátima Galiana Castelló, Department of Translation and Language Sciences, Pompeu Fabra University, Barcelona, Spain. Teun A. van Dijk, Department of Translation and Language Sciences, Pompeu Fabra University, Barcelona, Spain. Maria Alice de Mattos Pimenta Parente, Department of Developmental Psychology, Universidade Federal do Rio Grande do Sul (UFRGS), Porto Alegre, RS, Brazil. Jordi Peña-Casanova, Department of Behavioral Neurology. Service of Neurology, Hospital del Mar (IMAS) \& Behavioral Neurology Research Group, Program of Neuropsychopharmacology, Municipal Institute of Medical Research (IMIM). Correspondence regarding this article should be directed to: Lenisa Brandão, Núcleo de Estudos em Neuropsicologia Cognitiva, Instituto de Psicologia - Universidade Federal do Rio Grande do Sul, Rua Ramiro Barcelos, 2600, Santa Cecília, Porto Alegre, RS, Brazil, E-mail: lenisa.brandao@ufrgs.br unorganized and empty. Uninformative speech, a great number of indefinite terms, meaningless sentences and the absence of relevant elements for the comprehension of the message expressed can be observed as the disease progresses (Dijkstra, Bourgeois, Allen, \& Burgio, 2004; Dijkstra, Bourgeois, Petrie, Burgio, \& Allen-Burge, 2002; Laine, Laakso, Vuorinen, \& Rinne, 1998; Ripich \& Terrel, 1988). All these features demonstrate that AD produces a breakdown in discourse production processes at a semantic level. Although researchers agree that discourse meaning is clearly impaired, it is probably not due to the disruption of semantic memory alone. Deficits in episodic memory and working memory probably have a great impact in the manifestation of discourse disorders in $\mathrm{AD}$ (Ash, Moore, Vesely, \& Grossman, 2007). The need for understanding the possible prognosis of discourse interventions in $\mathrm{AD}$ directs our quest to the study of the processes behind successful use of informative prompts by persons with AD. The impairment of cognitive systems is likely to have a fundamental role in the compensation of discourse deficits. Understanding the links between cognition and discourse in $\mathrm{AD}$ can be useful not only for intervention purposes, but also for understanding the processes that underlie discourse production. 
According to van Dijk (2006), discourse production is a cognitive and partially conscious process that consists of the construction, allocation and update of mental representations of events and of the context of communication (mental and context models). The selection of relevant information from these representations and from general knowledge is the departure point of the process of discourse production. The activation of relevant knowledge in communicative situations as well as the interrelation and organization of types of knowledge in memory systems are crucial to explain the construction of coherent structures in discourse. Therefore, difficulties in the production of coherent discourse such as observed in Alzheimer's disease may arise from the breakdown or disruption in the access of mental models and world knowledge.

Mental models of events are cognitive representations of experiences, events and situations in episodic memory. Personal experiences are stored in autobiographical memory, which contains both specific and general autobiographical information. Episodic knowledge must interact with semantic knowledge in the process of representation of mental models. Semantic memory contains knowledge of the world, which enables reference to information stored in long-term memory and provides inherent knowledge of situations. This knowledge is common to all members pertaining to a society or culture (van Dijk, 2008).

In coherent discourse production, language users set out from a significant mental model and organize experiences into global semantic representations which enable identification of the topic of discourse. One of the main tasks in the process of discourse production is to carry out a global plan in a dynamic manner. This plan is represented by the macrostructure of discourse. Macrostructures are formed by the topic and the essential content of discourse, which are the most relevant ideas. The whole set of discourse propositions forms the microstructure. Propositions are generated through semantic strategies that regulate the recovery, construction and update of the set of proposals in the discourse (van Dijk \& Kintsch, 1983).

In the current study, two typical types of discourse features in $\mathrm{AD}$ are examined, incomplete propositions and repeated propositions. The production of incomplete propositions may signal a difficulty in accessing episodic information or in representing the semantic structure of discourse at the propositional level. The repetition of propositions may relate to difficulties in retrieving new events from a mental model, or in the semantic representation of new ideas. Both problems may lead to the non progression of discourse and persistence of previously produced ideas. It is likely that the persistence of ideas in $\mathrm{AD}$ does not have an involuntary nature, as in the case of perseverations (Blanken, Dittman, Haas \& Wallesch, 1987); rather, it can consist of a compensatory strategy used in order to keep the floor in conversation (Cruz, 2008).
Studies have shown that discourse deficits appear in $\mathrm{AD}$ in an autobiographical narrative, which is usually elicited by tasks in which participants receive a minimum of input from their communicative partner (Ash et al., 2007; Laine, Laakso, Vuorinen, \& Rinne, 1998; Ripich \& Terrel, 1988). The lack of informative input provides a necessary baseline for discourse performance in $\mathrm{AD}$, demonstrating how this population manages to produce discourse independently. These traditional neutral input tasks are also more common in research and clinical contexts because the examiner does not share autobiographical knowledge with the participant. However, the adoption of different methodological approaches may offer the possibility to study the use of compensation strategies that could help participants activate knowledge from mental models and represent this knowledge in a semantic level. What occurs with discourse in $\mathrm{AD}$ if the examiner knows crucial information about the event being told and provides this information when necessary? Which role does cognition play in the successful use of this information?

The current study compares the expression of incomplete and repeated propositions in a noninformative prompted task and in an informative prompted task. The analysis of context in this study is limited to the type of prompt provided in the tasks. In the non-informative prompted task, the examiner provides no information about the autobiographical event told by the participant. In the informative prompted task, crucial episodic information is provided according to the need of the participant. Neuropsychological data is used to examine if cognitive deficits can explain the presence of incomplete and repeated propositions in each task. The discussion debates on the role of cognition in the use of information provided in the context of communication.

\section{Method}

\section{Participants}

Groups with Alzheimer's disease (AD) Participants with AD consisted of 8 older adults in stage 4 (moderate cognitive decline) and 10 older adults in stage 5 (moderate-severe cognitive decline) according to the Global Deterioration Scale (GDS) (Reisberg, Ferris, de Léon, \& Crook, 1982). They had 4 to 8 years of education and were patients in the Neuropsychology Service of Hospital del Mar (Barcelona, Spain). The patients had been medically evaluated, and diagnosed as having $\mathrm{AD}$, based on neurological and behavioral findings (for a review of the methods used, see Robles, Alom, \& PeñaCasanova, 2002). Results of the screening with the Mini-mental State Exam (MMSE) (Folstein, Folstein, \& McHugh, 1975; Blesa et al., 2001), along with a more complete neuropsychological profile of the groups can be seen in Table 1. Disease 
stages were classified according to cognitive decline by means of the Global Deterioration Scale (GDS), which enabled the selection of patients in stages 4 and 5 . Stages 4 and 5 of the GDS also represent Obler's classification for language decline in Alzheimer's disease (Obler, 1983, personal communication), consisting of early and moderate stages of language disorders, respectively. Early stage (GDS 4) is characterized by a mild naming disturbance and incoherent discourse, with generally intact comprehension and pragmatic abilities. Language hallmarks of the moderate stage (GDS 5) are increased semantic impairment, with mild syntactic and phonologic errors (Obler, 1983, personal communication).

An additional group of 18 older adults, consisting of volunteers who were members of a parish and of a civic center, were selected to match the $\mathrm{AD}$ group in age and years of education and screened to exclude neurological and psychiatric pathologies. Two of these individuals were excluded. The 16 control subjects were interviewed and reported being in good health, with no history of speech, language or hearing problems. Controls also met the MMSE criteria (above 23/24 scores) for exclusion of dementia.

\section{Social and cultural features of the samples}

The Kruskal-Wallis statistical test did not detect significant differences between groups concerning age and education. The AD group had a medium of 79.83 $(\mathrm{SD}=3.63)$ years of age, while the control group had a medium of $79.50(S D=3.20)$ years. Concerning years of education, the AD group had $5.5(\mathrm{SD}=2.91)$ years, while the control group had $7.37(\mathrm{SD}=3.07$ ) years of education. The chi-square test also did not find significant differences between groups concerning gender and first language. In the AD group, 83.33\% were women, and in the control group, $81.25 \%$ were women. Concerning first language, in the $\mathrm{AD}$ group $50 \%$ had Spanish as their first language and the other $50 \%$, Catalan. In the control group, Spanish was the first language of $56.25 \%$ of the participants, while Catalan was the first language of $43.75 \%$ of them.

\section{Accompanying family members}

Accompanying family members were asked to tell two personal stories from the participant's life in order to provide a basis of information for the narrative prompts that would be given during the informative prompted discourse task with the patient. All participants and accompanying family members participated as volunteers, and a letter of consent that explained the purpose of the study was signed by the participant and by the responsible member from the family. The purpose of the study and ethical procedures, such as identity preservation, were explained.

\section{Neuropsychological evaluations}

To evaluate semantic memory, we used the Pyramids and Palm Threes Test (Gudayol-Ferré, 2000; Howard \& Patterson, 1992); to assess comprehension, the Token Test (De Renzi \& Faglioni, 1978); to test the lexicalsemantic systems, the Boston Naming Test (Kaplan, Goodglass, \& Weintraub, 2001); for short-term memory, the Digit Span (Peña-Casanova, 1990; Wechsler, 1997) and for working memory, the Digit Ordering Ttask (McDonald, Almor, Henderson, Kempler, \& Andersen, 2001). Episodic memory evaluations included the Autobiographical Memory Interview (Kopelman, 1994) and the prose memory task from the Barcelona Test (Peña-Casanova, 1990).

\section{Procedure}

Controls underwent a session of one hour and thirty minutes and AD groups underwent two sessions of one hour each. In the first part, personal narratives were audio taped. In the second part, participants underwent neuropsychological evaluations. Previous to the participants' discourse evaluation, accompanying family members of $\mathrm{AD}$ participants were interviewed individually. They were asked to tell two stories from the participant's lives that were thought to be remembered. The narratives told by the family member were used to extract the following information: (1) an interesting event or incident that happened in the patient's life, (2) the place where it happened, (3) the life time period when it took place, (4) the names of the persons in the story and their relation to the participant, and (5) the resolution of the story. The items of information were used as informative prompts in the informative prompted discourse task.

\section{Discourse tasks}

Topic-directed interviews took place in a quiet room, and the participants were assessed individually. All of the interviews were audio taped, and all of the interviewees were aware of the fact of being taped.

\section{(1) Non-informative prompts}

Wedding stories told during the AMI (youth period) were selected to analyze discourse performance in a communication situation in which no information about the story would be provided by the examiner. The wedding theme was selected due to high scoring of autobiographical memory. The instruction consisted in asking participants to tell a story related to their wedding. The examiner also used verbal and non-verbal signs of attention and support (such as bouncing the head and saying yes and umhum). Non-informative prompts consisted in: (1) general prompts for the recovery of an autobiographical event, given when the participant did not recall any event to tell on that topic (for example, something that happened to a guest); (2) continuity 
prompts were given during long pauses and whenever there was an early story interruption (for example, what else can you tell me?); and (3) topic return prompts were given whenever the participant changed topic abruptly (for example, so, lets go back to the wedding topic).

\section{(2) Informative prompts}

In this task, informative prompts were given after the patient's free expression of the story, at a second attempt to obtain the narrative proposed. Since memory of events are stored as mental models in episodic memory (Johnson-Laird, 1983; van Dijk, 2006), it is expected that information from the main schematic categories of such mental models - setting (time, place), participants (and their identities, roles and relations), main action and consequence (complication and resolution) - can serve as prompts to help participants tell their stories. Prompts were given only in the cases in which discourse lacked more than one category of the narrative superstructure (van Dijk, 1980), such as subcategories of setting and/or action progression, when expression of the story was too simplified and reduced or presented excessive repetitions and descriptions. Each of the orderly given prompts were provided one at a time by the examiner, during the patients`long pauses (longer than 3 seconds).

Discourse analysis - Transcripts and propositional analysis

Each interview was transcribed in its entirety, both orthographically and verbatim. Contextual notes, such as important gestures and other nonverbal behaviors that affected the comprehension of the story, were reported. Unintelligible utterances were excluded from the analysis. All sentences were analyzed in terms of their propositions.

A proposition consists of the predicate, and its argument(s). The predicate is usually expressed by a verb or an adjective. Arguments are usually expressed by nouns and present certain roles, such as agent, person who experiences, theme, recipient, instrument, time, location, source, goal, destination, manner and means (Saeed, 2003).

\section{Discourse variables}

Each of the following discourse variables were counted as instances which were added and divided by the total number of propositions expressed.

a) Incomplete propositions - These were propositions which were clearly missing an element, such as the predicate or an argument, affecting its comprehension.

Example:

In Spanish: [Fuimos allí a comer] y por la tarde... cambio de piso... en una galería muy grande y [allí pues a bailar todo el mundo].

In English: [We went there to eat] and in the afternoon... change of apartment... in a very large gallery and so [there everybody danced].

In the example above, a participant in the moderatesevere stage tries to narrate her wedding, but only succeeds is expressing two complete propositions. The text in bold shows what seems to be the expression of an incomplete idea.

b) Repeated propositions - These were instances of ideas which were repeated without having a useful role for the comprehension of the story.

Example:

In Spanish: [En la fiesta se comía estupendamente] y [fue con muchas amigas de allí del colegio]... si... y [vinieron mis suegros y mi cuñada] y si... [muy bien] [muy bien] [muy bien] [eso muy bien] [todo muy bien].

In English: [At the party you could eat wonderfully] and [there were many friends from school] ... and ... [my in-laws came and my sister in law] and yes ... [very well] [very well] [very well] [this went well] [everything went well].

In this example, a participant in the early stage of $\mathrm{AD}$ repeats the idea that her wedding party went well five times.

\section{Reliability}

A random part of the transcripts (15\%) was taken to calculate the agreement between the two independent judges, using Kendall's Tau statistical test. For incomplete propositions the agreement

Table 1. Neuropsychological profile of participants. Mean and standard deviation values accompanied by different letters indicate that significant differences were detected $(\mathrm{p}<.05)$ with the Kruskal-Wallis test. The AMI assessed personal semantic (in the table, semantic) and autobiographical (in the table, incident) knowledge. Textual memory assessed free recall (in the table, free) and prompted recall (in the table, prompted).

\begin{tabular}{|c|c|c|c|c|c|c|c|c|c|c|}
\hline \multirow[b]{2}{*}{ Group } & \multirow[b]{2}{*}{ MMSE } & \multirow{2}{*}{$\begin{array}{l}\text { Token } \\
\text { Test }\end{array}$} & \multirow{2}{*}{$\begin{array}{c}\text { Pyramids } \\
\text { and Palm } \\
\text { Threes } \\
\text { Test }\end{array}$} & \multirow{2}{*}{$\begin{array}{c}\text { Boston } \\
\text { Naming } \\
\text { Test }\end{array}$} & \multirow{2}{*}{$\begin{array}{l}\text { Digit } \\
\text { Span }\end{array}$} & \multirow{2}{*}{$\begin{array}{c}\text { Digit } \\
\text { ordering }\end{array}$} & \multicolumn{2}{|c|}{ AMI } & \multicolumn{2}{|c|}{ Prose memory } \\
\hline & & & & & & & Semantic & Incident & Free & $\begin{array}{c}\text { Promp- } \\
\text { ted }\end{array}$ \\
\hline $\begin{array}{l}\text { Con- } \\
\text { trols }\end{array}$ & $\begin{array}{c}28.75 \\
(1.12) a\end{array}$ & $\begin{array}{c}32.65 \\
(2.24) a\end{array}$ & $\begin{array}{c}49.81 \\
(1.60) a\end{array}$ & $\begin{array}{c}44.43 \\
(4.56) a\end{array}$ & $\begin{array}{c}5.43 \\
(.62) a\end{array}$ & $\begin{array}{c}5.06 \\
(.85) a\end{array}$ & $\begin{array}{c}53,81 \\
(3.02) a\end{array}$ & $\begin{array}{c}24.06 \\
(1.65) a\end{array}$ & $\begin{array}{c}14.90 \\
(2.85) a\end{array}$ & $\begin{array}{c}17.93 \\
(1.97) a\end{array}$ \\
\hline GDS 4 & $\begin{array}{c}22.50 \\
(4.40) b\end{array}$ & $\begin{array}{c}24.12 \\
(8.85) b\end{array}$ & $\begin{array}{c}43.21 \\
(6.54) b\end{array}$ & $\begin{array}{c}29.00 \\
(7.46) b\end{array}$ & $\begin{array}{l}4.50 \\
(.92) b\end{array}$ & $\begin{array}{c}3.00 \\
(2.20) b\end{array}$ & $\begin{array}{c}39.18 \\
(16.09) b\end{array}$ & $\begin{array}{c}15,50 \\
(7.72) b\end{array}$ & $\begin{array}{c}6.06 \\
(2.93) b\end{array}$ & $\begin{array}{c}8.93 \\
(4.44) b\end{array}$ \\
\hline GDS 5 & $\begin{array}{l}16.10 \\
(2.18) c\end{array}$ & $\begin{array}{c}17.00 \\
(5.29) c\end{array}$ & $\begin{array}{c}30.50 \\
(7.48) c\end{array}$ & $\begin{array}{c}21.30 \\
(4.98) C\end{array}$ & $4.1 \underset{b}{(.87)}$ & $\begin{array}{l}0.60 \\
(.96) c\end{array}$ & $\begin{array}{c}25.85 \\
(10.11) c\end{array}$ & $\begin{array}{l}10.80 \\
(6.21) b\end{array}$ & $\begin{array}{l}2.60 \\
(2.01) c\end{array}$ & $\begin{array}{l}3.70 \\
(2.97) c\end{array}$ \\
\hline
\end{tabular}


obtained was .81 and for repeated propositions the agreement obtained was .85 .

\section{Results}

\section{Neuropsychological profile of the groups}

According to the Mann-Whitney test, the AD sample differed significantly $(p<.001)$ from the control group in all neuropsychological scores. All evaluations yielded significant differences between groups, except the Digit Span and the Autobiographical Memory for incidents test.

\section{Differences between groups}

As displayed in Table 2, the Kruskall-Wallis test showed that differences in terms of repetitions were not significant when calculated over the total number of propositions (proportions). In contrast, $\mathrm{AD}$ groups differed significantly from the control group in relation "repeated propositions" was not sufficiently expressive to yield significant differences between control and AD groups. This result may well be explained by the smaller sample of participants, a limitation of the current study. Differences concerning repeated ideas were observed only when absolute numbers were compared and not when proportions were calculated over the number of ideas produced. Tomoeda \& Bayles (1993) also observed that when taken as absolute numbers, the repetition of ideas differed even between participants with early and moderate $\mathrm{AD}$, with more repetitions in the early stage of the disease. Considering these findings, we question whether the presence of repeated ideas is a deficit marker or a strategy used to compensate for difficulties in the production of new ideas. Despite being regarded as a sign of perseveration by some authors (Tomoeda \& Bayles, 1993), repetitions produced by speakers with $\mathrm{AD}$ are considered distinct from perseverations observed

Table 2. Differences between groups. Mean and standard deviation values accompanied by different letters represent significant difference between groups $(p<.05)$.

\begin{tabular}{|c|c|c|c|c|}
\hline \multirow{2}{*}{ Discourse variables } & \multirow{2}{*}{ Prompted task } & GDS 4 & GDS 5 & Controls \\
\hline & & Mean (SD) & Mean (SD) & Mean (SD) \\
\hline \multirow[t]{2}{*}{$\begin{array}{l}\text { Incomplete } \\
\text { propositions }\end{array}$} & Non informative & 2.50 (2.61)a & 2.30 (2.35)a & $.43(1.20) \mathrm{b}$ \\
\hline & Informative & 1.28 (2.98)a & $4.00(4.00) \mathrm{a}$ & - \\
\hline \multirow{2}{*}{$\begin{array}{l}\text { Repeated } \\
\text { propositions }\end{array}$} & Non informative & $1.00(1.41) \mathrm{a}$ & 1.40 (1.83)a & $.12(.34) \mathrm{a}$ \\
\hline & Informative & $0.14(.37) \mathrm{a}$ & .60 (.89)a & - \\
\hline
\end{tabular}

to the proportion of incomplete propositions in the noninformative prompt task.

\section{Correlations with cognitive variables (AD group)}

As shown in Table 3, negative correlations were found between the proportion of incomplete propositions produced in the informative prompt task and scores from the Token Test $(\rho=-.90 ; p<.005)$, Pyramids and Palm Threes Test $(\rho=-.79 ; p<.005)$, Digit Span $(\rho=-.79 ; p$ $<.01$ ), semantic-autobiographic knowledge from AMI $(\rho=-.77 ; p<.01)$, Digit Ordering $(\rho=-.64 ; p<.05)$ and Boston Naming Test $(\rho=-.58 ; p<.05)$.

\section{Discussion}

The current study investigated two common discourse measures in studies about discourse deficits in $\mathrm{AD}$. In contrast with previous studies (Blanken et al., 1987; Cruz, 2008), we concluded that the variable in aphasic discourse (Blanken et al., 1987). When persons with $\mathrm{AD}$ repeat ideas or words, these are mostly on-topic, justifying the use of the term soft repetitions (Blanken et al., 1987). Additionally, a greater discourse corpus would enable a classification of repetitions. Cruz (2008) showed that speakers with AD produce different types of repetitions, with specific functions within social interaction: some seem to have a structural and monitoring role in conversation; others can be related to the degenerative and progressive character of the sociocognitive loss in AD. According to Cruz (2008), when repetition is considered to have a strategic role in Alzheimer's speech, it could bring to light whether or not people with $\mathrm{AD}$ are able to manage knowledge in conversation. Moreover, proposition repetition may have many other reasons or causes, for instance rhetorical ones (persuasion), didactic ones (explanation), narrative ones (emotion, etc.). The different roles repetition may play might be the underlying explanation to why the 
Table 3. Correlations between discourse and cognitive measures. *Represents Spearman correlations with $\mathrm{p}<.05 ;{ }^{* *}$ represent significant Spearman correlations with $\mathrm{p}<.01$.

\begin{tabular}{|c|c|c|c|c|}
\hline \multirow{2}{*}{ Discourse and cognitive measures } & \multicolumn{2}{|c|}{ Non informative prompted task } & \multicolumn{2}{|c|}{ Informative prompted task } \\
\hline & $\begin{array}{l}\text { Incomplete } \\
\text { propositions }\end{array}$ & $\begin{array}{c}\text { Repeated } \\
\text { propositions }\end{array}$ & $\begin{array}{c}\text { Incomplete } \\
\text { propositions }\end{array}$ & $\begin{array}{c}\text { Repeated } \\
\text { propositions }\end{array}$ \\
\hline MMSE & -.20 & .01 & $-.83 * *$ & .08 \\
\hline Token Test & -.33 & -.18 & $-.90 * *$ & .03 \\
\hline Boston Naming Test & -.01 & .13 & $-.58^{*}$ & .02 \\
\hline Pyramids and Palm Threes & -.03 & .22 & $-.79 * *$ & -.05 \\
\hline Digit span & -.41 & -.28 & $-.79 * *$ & .12 \\
\hline Digit ordering & -.37 & -.12 & $-.64^{*}$ & .04 \\
\hline Free recall prose memory & -.29 & -.02 & $-.82 * *$ & .04 \\
\hline Prompted prose memory & -.30 & .22 & $-.84 * *$ & -.01 \\
\hline AMI semantic & .05 & .30 & $-.77 * *$ & -.25 \\
\hline AMI incident & -.08 & .17 & -.50 & -.45 \\
\hline
\end{tabular}

current study did not find significant results related to the repetition of ideas that could point to a possible discourse deficit or strategy.

The fact that $\mathrm{AD}$ groups differed significantly from the control group in relation to the proportion of incomplete propositions in the non-informative prompted task suggests that this specific discourse variable is of special interest, as it may be a sensitive measure to detect AD. Earlier studies (Glosser \& Deser, 1990; Ripich \& Terrell, 1988) also demonstrated that the amount of missing elements in discourse elicited without informative prompts is specially related to discourse incoherence in $\mathrm{AD}$. A greater number of incomplete propositions are produced when speakers with $\mathrm{AD}$ strive to produce their narrative independently.

One of the limitations of the current study was the lack of comparison with controls in the informative prompted task. This task was not undertaken by controls due to the limited access that the examiner had to family members of controls. Alternatively, a different analysis was later conducted in order to answer the following question: would the number of incomplete propositions produced by participants with $\mathrm{AD}$ when given informative prompts differ from the number of incomplete propositions expressed by controls when not given informative prompts? This question was addressed in an attempt to verify if informative prompts can be used efficiently to decrease the expression of incomplete ideas by persons with $\mathrm{AD}$. If the number of incomplete propositions produced in the prompted situation would not differ from that expressed by older adults without dementia in a non-prompted situation, then informative prompts would be a recommendable compensation strategy to use for persons with AD. This was actually true for participants in the moderate stage (GDS 4) of $\mathrm{AD}$, whose proportions of incomplete propositions did not differ significantly from controls $(U=47.00$; $\mathrm{p}=.57)$. In contrast, participants in the moderatesevere stage (GDS 5) were not able to compensate by means of the use of informative prompts, expressing significantly more incomplete ideas than controls $(\mathrm{U}=$ $13.00 ; p<.05)$. The inability to use informative prompts in compensating for incomplete propositions seems to reflect impairment in knowledge management in the later stages of the disease. In this sense, discourse tasks which provide a context in which external information is given can be particularly useful to detect knowledge management problems in this population. 
The production of incomplete propositions may mean that the underlying mental models of events are fragmentary. For instance, persons with AD may remember that someone visited them this morning, but not who it was. Or conversely, that someone was there this morning, but one does not remember what he or she did. Similarly, setting information may be missing, for example, where and when something happened. The fragmentation of mental models would provide a macrolinguistic explanation to incomplete propositions. In this case, the disrupted level causing the expression of incomplete propositions would have a much greater relation with problems in episodic memory.

Our results concerning correlations of incomplete propositions with episodic memory measures point to a strong negative correlation with the prose (logical) memory task, for both free and prompted recall scores (Table 3). This means that participants with lower scores on prose recall expressed more incomplete propositions. Nevertheless, considering that the discourse tasks used elicited personal narratives, the most relevant episodic measure for this study would be the AMI scores. Autobiographical memory scores demonstrated that the type of autobiographic knowledge that correlated to the number of incomplete propositions in the informative promptedtaskwassemanticautobiographicalknowledge. This might mean that the lack of knowledge of facts about one's own life, such as the name of the church where one's wedding took place, has a greater impact in the expression of incomplete ideas than specific knowledge from the wedding episode (mental model). In the informative prompted task, participants received specific information about the event (place, time, participants, complication and resolution). It can be inferred that the prompted superstructure elements of mental models provided specific episodic information, compensating for the possible fragmentation of the incident narrated. This could explain why autobiographical memory scores for incidents did not correlate with the expression of incomplete propositions. Instead, the negative correlation with semantic autobiographical scores showed that expression of incomplete propositions increased as the fragmentation of general knowledge about life events decreased. Semantic knowledge about personal events is more preserved (view the neuropsychological profile of participants in Table 1) than memory for autobiographical incidents. Perhaps this means that when there were difficulties in accessing factual knowledge about events, more incomplete ideas appeared in discourse.

The notion that the disruption of knowledge of the semantic type would have a greater impact in the expression of incomplete ideas seems to be confirmed by its strong correlations with semantic memory measures. The consensus view in cognitive psychology is that language comprehension is an integral part of the construction of mental models. The strongest correlation observed between incomplete propositions in the informative prompted task and cognitive measures was the correlation with Token test scores. This test is designed to assess verbal comprehension of commands of increasing complexity. To understand verbal commands participants basically had to find meaning in what was requested, that is, relate ideas to their world knowledge. Impairment of the semantic system was highly associated with the expression of incomplete propositions when informative prompts were given. This means participants with $\mathrm{AD}$ probably had problems understanding the prompts, that is, they could not build meaningful relations between the prompts and their knowledge of events. Thus, they expressed incomplete semantic representations (propositions) of discourse.

At the microlinguistic level (Levelt, Meyer, \& Roelofs, 2004), it could be argued that the production of incomplete propositions does not necessarily mean a problem in episodic or semantic representation, but a (surface) grammar difficulty to express propositions in grammatical clauses and sentences. This latter explanation of incomplete propositions is not considered suitable, as even participants in the moderate-severe stage of AD still had relatively preserved grammatical skills.

The high number of correlations observed between cognitive scores and incomplete propositions in the informative prompted task (rather than in the noninformative prompted task) seems to be the most interesting result of this study. It is inferred that the expression of incomplete propositions in the informative prompted task reflects a failure in knowledge management. If the number of incomplete propositions would have also correlated with the cognitive scores in the non-informative prompted task, then this discourse deficit would seem to manifest independently from the interaction with information provided in the context. If this was the case, the expression of incomplete ideas would simply relate to cognitive decline. Instead, the correlations were observed only in the informative prompted task. This finding clearly suggests that the ability to successfully manage knowledge given in the context relies heavily on the preservation of memory systems.

The speaker can not succeed in manipulating information given by his/her interlocutor if semantic and episodic memories are not sufficiently preserved. In other words, the use of relevant information from the context can only take place if the speaker is able to find meaningful connections between the information from the context and his own stored knowledge. As the correlations with short-term memory show (Table 3), this memory system also plays a role in storing given information and a well start up of working memory is needed in order to establish a reference frame and to maintain the main focus of attention in a dynamic manner (Almor, Kempler, MacDonald, Andersen, \& Tyler, 1999; Kintsch \& van Dijk, 1978). Only in such 
conditions can the speaker use information provided by his or her interlocutor to build an on-line coherent representation of discourse.

Our results support previous research findings on the role of the communicative context in the interplay between discourse and cognition, that is, its influence in the improvement and compensation of communicative difficulties in AD (March, Pattison, \& Wales, 2009). However, it is important to point out that the correlation findings reported here are not aimed to tease out the exact relationship between knowledge management and cognition. The results rather show that the role of cognition in discourse production is closely linked to the capacity of managing information provided in the context of communication.

\section{Conclusions}

The main findings of the current study demonstrated that the expression of incomplete propositions is an important variable in the study of discourse in AD. The performance of moderate stage AD participants showed that this discourse feature is sensitive to compensation by means of the use of informative prompts. This finding is a promising one concerning the possibility of its use in interventions focused in improving and maintaining communication skills in AD. Negative correlations between the expression of incomplete propositions and cognitive scores suggested that successful compensation through informative prompts is mediated by the preservation of episodic and especially semantic skills. These results support the idea that the ability to manage episodic knowledge provided during discourse production depends largely in the preservation of comprehension and memory. Future studies should further explore the relations between discourse and cognition and the use of compensation strategies in different contexts of communication.

\section{Acknowledgments}

We would like to thank the Brazilian research funding organization CAPES for the one-year PhD scholarship that financed the first author to study in Barcelona in 2003. Our special thank you to the participants and their family members for the time and the energy spent in the study. We are also grateful to the Parish of the Church Sant Miquel del Port and to the Space for older people (Espai per a gent gran) at the Civic Center of Ateneu Fort Pienc, for the divulgation and for allowing us to use space for interviews with controls. Last but not least, we thank the Neuropsychology Service of Hospital del Mar (Barcelona, Spain) and the researchers who performed the role of judges for the discourse agreement analysis.

\section{References}

Almor, A., Kempler, D., MacDonald M., Andersen E., \& Tyler, L. (1999). Why do Alzheimer patients have difficulty with pronouns? Working memory, semantics, and reference in comprehension and production in Alzheimer's disease. Brain Language, 67, 202-227.

Ash, S., Moore, P., Vesely, L., \& Grossman, M. (2007). The decline of narrative discourse in Alzheimer's disease. Brain and Language, 103, 248-249.

Blanken, G., Dittman, J., Haas, J-C., \& Wallesch,C-W. (1987). Spontaneous speech in senile dementia and aphasia. Implications for a neurolinguistic model of language production. Cognition, 27, 247-274.

Blesa, R., Pujol, M., Aguilar, M., Santacruz, P., Bertran-Serra, I., Hernández, G., Sol, J.M., Peña-Casanova, J., \& NORMACODEM Group (2001). Clinical validity of the "mini-mental state” for Spanish speaking communities. Neuropsychologia, 39(11), 1150-1157.

Cruz, F. (2008). Linguagem, Interação e Cognição na Doença de Alzheimer. Unpublished Doctoral Thesis. UNICAMP, São Paulo, Brazil.

De Renzi, E., \& Faglioni, P. (1978). Normative data and screening power of a shortened version of the Token Test. Cortex, 14, 41-49.

Dijkstra, K., Bourgeois, M.S., Allen, R.S., \& Burgio, L.D. (2004). Conversational coherence: Discourse analysis of older adults with and without dementia. Journal of Neurolinguistics, 17, 263-283.

Dijkstra, K., Bourgeois, M., Petrie, G., Burgio, L., \& Allen-Burge, R. (2002). My recaller is on vacation: Discourse analysis of nursing home residents with dementia. Discourse Processes, 33, 55-76.

Folstein M.F., Folstein, S.E., \& McHugh, P.R. (1975). Mini-Mental State. A practical method for grading the cognitive state of patients for the clinician. Journal of Psychiatric Research, 12, 189-198.

Glosser, G., \& Desser, T. (1990). Patterns of discourse production among neurological patients with fluent language disorders. Brain and Language, 40, 67-88.

Gudayol-Ferré, E. (2000). Normalización del Test de Pirámides y Palmeras en una población española. Unpublished Master Theses: Universitat Autónoma de Barcelona.

Howard, D., \& Patterson, K. (1992). The Pyramids and Palm Trees Test. Berkshire: Thames Valley Test Company.

Johnson-Laird, P.N. (1983). Mental models: Towards a cognitive science of language, inference, and consciousness. Cambridge, MA: Harvard University Press.

Kaplan, E., Goodglass, H., \& Weintraub, S. (2001). Boston Naming Test. Philadelphia: Lippincott Williams \& Wilkins.

Kintsch, W., \& Van Dijk, T. (1978). Toward a model of text comprehension and production. Psychological Review, 85(5), 363-383.

Kopelman, M.D. (1994). The autobiographical memory interview (AMI) in organic and psychogenic amnesia. Memory, 2, 211-235.

Laine, M., Laakso, M., Vuorinen, E., \& Rinne, J. (1998). Coherence and informativeness of discourse in two dementia types. Journal of Neurolinguistics, 11(1-2), 79-87.

Levelt, W., Meyer, A., \& Roelofs, A. (2004). Relations of Lexical Access to Neural Implementation and Syntactic Encoding. Behavioral and Brain Sciences, 27(2), 299-301.

MacDonald, M., Almor, A., Henderson, V., Kempler, D., \& Andersen, E. (2001). Assessing working memory and language comprehension in Alzheimer's Disease. Brain and Language, 78, 17-42.

March, E.G., Pattisson, P., \& Wales, R. (2009). The role of cognition in context-dependent language use: Evidence from Alzheimer's disease. Journal of_Neurolinguistics, 22, 18-36.

Peña-Casanova, J. (1990). Programa Integrado de Exploración Neuropsicológica Test Barcelona (Manual). Barcelona: Masson.

Reisberg, B., Ferris, S.H., de León, M.J., \& Crook, T. (1982). The global deterioration scale for assessment of primary degenerative dementia. American Journal of_Psychiatry, 139, 1136-1139.

Ripich, D.N., \& Terrell, B.Y. (1988). Patterns of discourse cohesion and coherence in Alzheimer's disease. Journal of Speech and Hearing Disorders, 53, 8-15.

Robles, A., Alom, D.S., \& Peña-Casanova, J. (2002). Propuesta de criterios para el diagnostico clinico del deterioro cognitivo ligero, la demencia y la enfermedad de Alzheimer. Neurología, 17,17-32.

Saeed, J.I. (2003). Semantics - introducing linguistics. Oxford: Blackwell Publishing Press.

Tomoeda, C.K., \& Bayles, K.A. (1993). Longitudinal effects of Alzheimer disease on discourse production. Alzheimer Disease and Associated Disorders, 7(4), 223-236.

Van Dijk, T.A. (1980). Macrostructures. An interdisciplinary study of 
global structures in discourse, interaction and cognition. Hillsdale, NJ: Erlbaum.

Van Dijk, T.A. (2006). Discourse, context and cognition. Discourse Studies, 8(1), 159-177. Van Dijk, T.A. (2008). Discourse and Context. A Sociocognitive Approach. Cambridge: Cambridge
University Press. Van Dijk, T.A, \& Kintsch, W. (1983). Strategies of Discourse Comprehension. London: Academic Press.

Wechsler, D. (1997). Wechsler Adult Intelligence Scale-Third Edition (WAIS-III) Administration and Scoring Manual. San Antonio, TX: The Psychological Corporation. 